\title{
The Sznajd Dynamics on a Directed Clustered Network*
}

\author{
K. MalarZ ${ }^{\dagger}$ And K. KuŁakowski \\ Faculty of Physics and Applied Computer Science \\ AGH University of Science and Technology \\ al. Mickiewicza 30, PL-30-059 Cracow, Poland
}

\begin{abstract}
The Sznajd model is investigated in the directed Erdős-Rényi network with the clusterization coefficient enhanced to 0.3 by the method of Holme and Kim. Within additional triangles, all six links are present. In this network, some nodes preserve the minority opinion. The time $\tau$ of getting equilibrium is found to follow the log-normal distribution and it increases linearly with the system size. Its dependence on the initial opinion distribution is different from the analytical results for fully connected networks.
\end{abstract}

PACS numbers: 07.05.Tp, 87.23.Ge, 89.20.-a, 89.65.-s, 89.75.-k

\section{Introduction}

As it was formulated by Woodrow Wilson, opinion ultimately governs the world [1]; the common interest in its research is then more than justified. The rule of the public opinion (PO) got a new dimension when the mass media appeared at the beginning of the 20th century. The change was recognized by Walter Lippmann who shaped our understanding of PO [2]. In social sciences, large effort is devoted to capture necessary ingredients of a qualitative description of PO [3]. However, the role of structure of the social network was also thoroughly investigated [4]. The main message from the social sciences is that the PO dynamics proceeds in a landscape of established identifications. Then, variations of PO are possible through local current reinterpretations of political events rather than through converting of large groups [5]. Important difference - the so-called pluralistic ignorance appears between the actual and the expressed opinion, and it is only the latter which influences the dynamics [6].

Physicists entered to the field via statistical mechanics [7]; a recent review on this kind of research of PO dynamics can be found in Ref. [8]. In these quantitative

${ }^{*}$ Dedicated to Dietrich Stauffer on his 65th birthday.

${ }^{\dagger}$ corresponding author; e-mail: malarz@agh.edu.pl 
models, main role is played by the structure of social networks. Actual shape of this structure is a matter of discussion [9] and simple solutions cannot be expected. However, various models have been and continuously are constructed to capture this or that feature of the structure of at least selected social groups. The voter model describes the opinion spreading via links of direct neighborhood $[10,11]$. In the Sznajd model [12-14] an accordance of two neighbors is necessary to influence other neighbors of the pair. In the Deffuant et al. model [15], attention is paid to the difference of opinion of the neighbors; if this is too large, the transfer of opinion is blocked. These efforts seem to complete a consistent path towards a realistic although model description of the PO dynamics within the social structure approximated by a network.

The aim of this work is to make next step in this direction. The choice we have to make is twofold: to select the dynamic rule and to select the structure. Here we use the Sznajd model; in our opinion this model gives a bridge from the Granovetter concept of threshold [16] to computer simulations. Here the threshold is set as two persons, which is an important generalization of the voter model and which allows to take into account the correlations of opinions of neighbors. The structure of our model network is random and clustered [17], it shows the smallworld property [17] and it contains directed links; the last condition comes from the observed asymmetry of social relations [18]. Below in the text, the construction of the network is described in detail.

Here we concentrate on the calculations of the time $\tau$ of getting a stationary distribution of opinions. In this final stage although local opinions can change, the average opinion calculated over the whole network remains constant. In political sciences, the initial support for a new government collapses in some time, which can be identified by $\tau$. To give examples from recent Polish history, the timescale of this fall seems to be about two years (Jerzy Buzek) or shorter (Leszek Miller) [19]. We note that our calculations do not capture the metastable states of PO, where the majority is silent and activates only during an open debate [6].

\section{The network and the model}

The model society is the directed network [18] based on the Erdös-Rényi random graph [20] with enhanced clusterization [21]. The primary network is constructed such that each node has exactly three outgoing links, i.e. $P_{\text {out }}(k)=$ $\delta_{k, 3}$, as presented in Fig. 1a. The second step in network preparation is a splitting of each node of the primary network into the well clustered set of three nodes presented in Fig. 1b. Other method of controling the network topology was presented in Ref. [22]. An example of the final network of $N=30$ nodes is presented in Fig. 1c.

The spin like opinion $s_{i}= \pm 1$ is assigned to each node/actor $i$. The opinion dynamics is governed by original Sznajd rule [12]: each time step we scan all $N$ nodes according to a random permutation of the nodes labels. For each investigated node $i$ one of its three out-neighbors $j$ is selected. If the opinions of such 


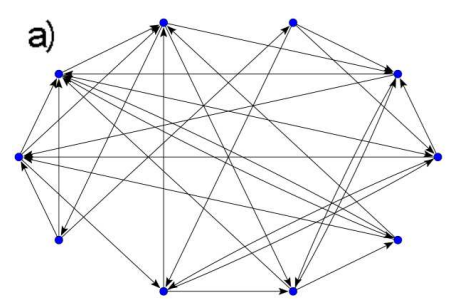

b)

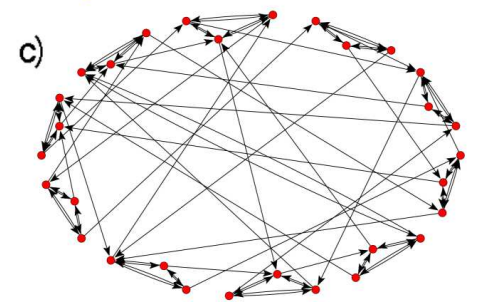

Fig. 1. Network construction. In both cases $P_{\text {out }}(k)=\delta_{k, 3} . N=30$.

selected pair are identical $\left(s_{i} s_{j}=1\right)$ then each out-neighbors of the pair accepts the pair's opinion with the probability $p$.

Initially, the fraction $p_{0}$ is of the negative opinion. The results of simulations are averaged over $N_{\text {run }}$ independent runs.

\section{Results}

For the Sznajd model, the system tends to the state of uniform opinion, i.e.

$$
m=\frac{1}{N} \sum_{i=1}^{N} s_{i}= \pm 1
$$

basing on the majority of the initial opinions. Here however, some actors will never change their initial opinion and full consensus may be never reached. To evaluate the number of such actors, let us consider a triangle of them presented in the right part of Fig. 1b. In this case, when all central spins have the same initial opinion, they will sustain it and will support each other in keeping it constant. The fraction of such triangles depends on the concentration of initial majority $p_{0}^{\mathrm{c}}=\frac{1}{2}+\left|p_{0}-\frac{1}{2}\right|$. Having this concentration, we can evaluate the number of triangles where at least two of three actors are of minority opinion. With the Sznajd evolution rule, two actors are expected to convince the third one. Of such triangles, a fraction $\exp (-3)$ have no in-neighbors according to the Poisson probability. Hence they will remain in their state. Instead of unity, the asymptotic value of the magnetization will be at most

$$
m_{\infty}=\left\{\begin{array}{l}
2 \mathrm{e}^{-3}\left(1+2 p_{0}^{\mathrm{c}}\right)\left(1-p_{0}^{\mathrm{c}}\right)^{2}-1 \quad \Longleftrightarrow p_{0}>0.5, \\
1-2 \mathrm{e}^{-3}\left(1+2 p_{0}^{\mathrm{c}}\right)\left(1-p_{0}^{\mathrm{c}}\right)^{2} \quad \Longleftrightarrow p_{0}<0.5 .
\end{array}\right.
$$

The true value of $\left|m_{\infty}\right|$ is found numerically. The simulation takes $N_{\text {iter }}$ steps 
(and in each time step $N$ actors pairs is investigated) unless the saturation opinion $\left(\left|m_{\infty}\right|<1\right)$ is reached earlier.

The time evolution of the average opinion $m$ for

- different values of initial fraction $p_{0}$ of one opinion,

- system size $N$,

- and probability of following Sznajd rule $p$,

are presented in Fig. $2 \mathrm{a}-\mathrm{c}$, respectively.

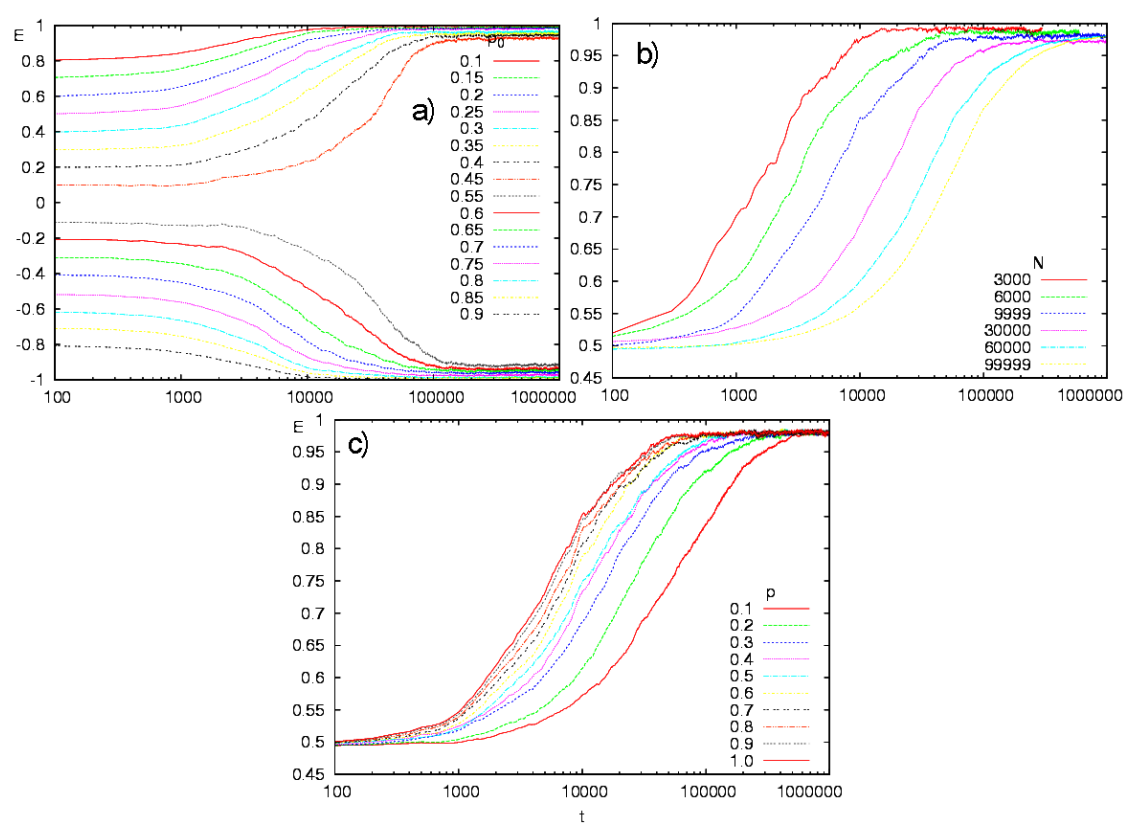

Fig. 2. Time evolution of average opinion dynamics for different values of (a) initial fraction $p_{0}$ of one of the opinion, (b) system size $N$ and (c) probability of following Sznajd rule $p$.

The average time $\tau$ of reaching saturation opinion $m_{\infty}$ depends:

- exponentially with difference $p_{0}$ on the critical concentration $1 / 2$ :

$$
\tau \propto \exp \left(\frac{-\left|p_{0}-1 / 2\right|}{\xi}\right)
$$

with $\xi=6.90$ (Fig. 3a),

- according to power law with the probability of following Sznajd rule $\tau \propto p^{-\gamma}$, $\gamma=0.723$ (Fig. 3c),

- and linearly with the system size $\tau=4.47 N+6703$ (Fig. 3b). 


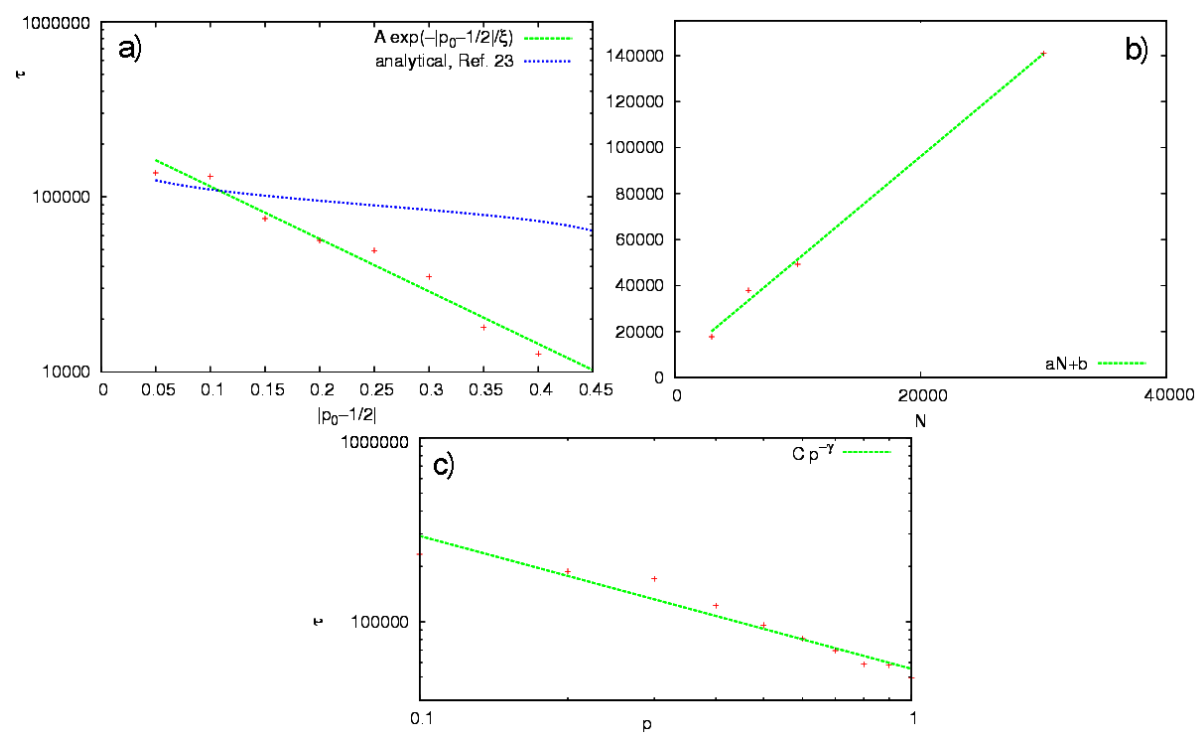

Fig. 3. Time $\tau$ of reaching the consensus for different values of (a) initial fraction $p_{0}$ of one of the opinion, (b) system size $N$ and (c) probability of the following Sznajd rule $p$, when the other parameters are fixed on $N=9999, p=1, p_{0}=0.25$. The fitted parameters are $\xi=6.90, \gamma=0.723, a=4.47, b=6703$.

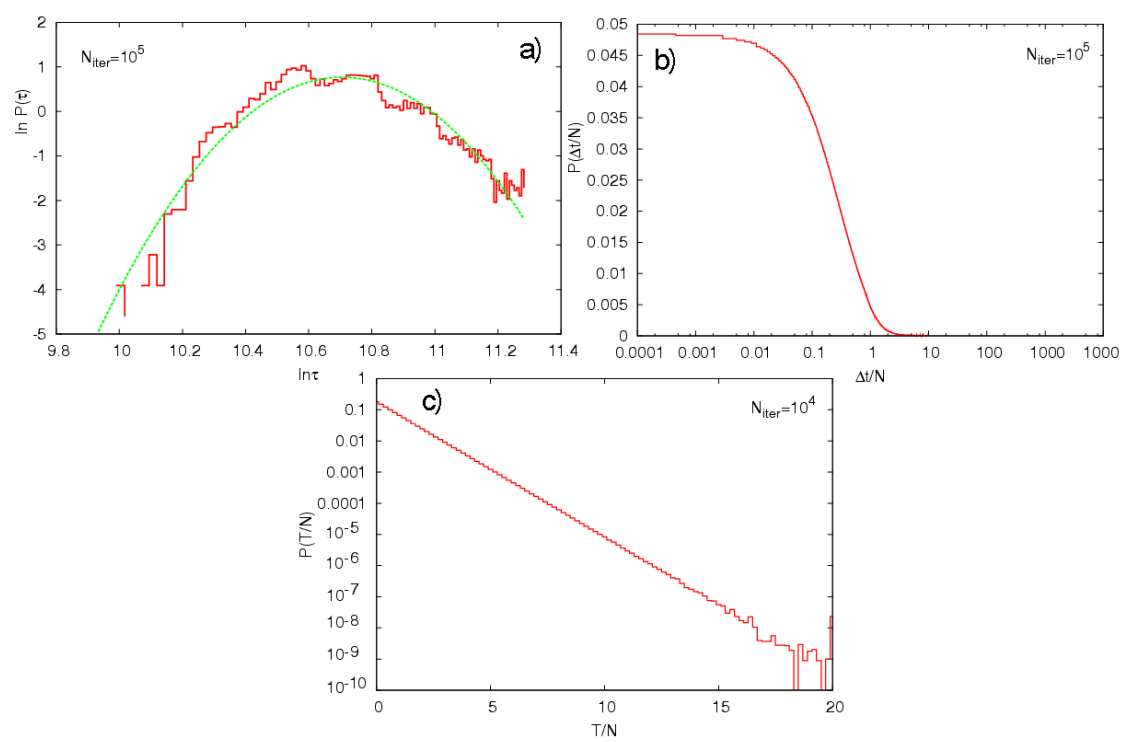

Fig. 4. The distribution of time (a) $\tau$ of reaching the consensus, (b) $\Delta t$ of delay between subsequent flips of the given spin, (c) $T$ of visiting the given spin. In the latter case the spins are visited in completely random fashion and not according the permutation of the spin label. $N=9999, p_{0}=0.25, p=1, N_{\text {run }}=100$. 
The last result was observed and explained earlier in Ref. [23] as well. The fact that $\xi<1$ is due to the fact that decreasing $p$, we change the order of updating spins from purely random one to the random permutation.

The distribution $P(\tau)$ of reaching the consensus time seems to be log-normal. An example of such distribution for $N_{\text {run }}=100$ independent simulations is shown in Fig. 4a. We have checked the distribution $P$ of times $\Delta t$ between subsequent flips of given spins and the one of times $T$ between reaching the same node. The distributions are presented in Figs. 4b and c. In the latter case nodes are selected in purely random fashion and not according to actors labels permutations.

\section{Discussion}

As remarked above, in our network some triangles have no in-links. These nodes and some of their neighbors remain in the initial state, and therefore full accordance of opinions cannot be reached. This is an important difference between our model and other simulations of the Sznajd dynamics.

The shape of the obtained curve on the mean opinion against time agrees qualitatively with the accessible experimental data [19] on the fall of the public support for the policy of the government of Jerzy Buzek on the social issues: social security and health protection between January 1998 and June 2001. This comparison is shown in Fig. 5. The curves on other issues are practically the same (agricultural policy), show the similar timescale (economy and management), or do not vary significantly (foreign affairs). We note however that in this version of the Sznajd model the majority always wins. Then the results on the fall of the public support can be compared only to those data, where the initial support was already smaller than fifty per cent.

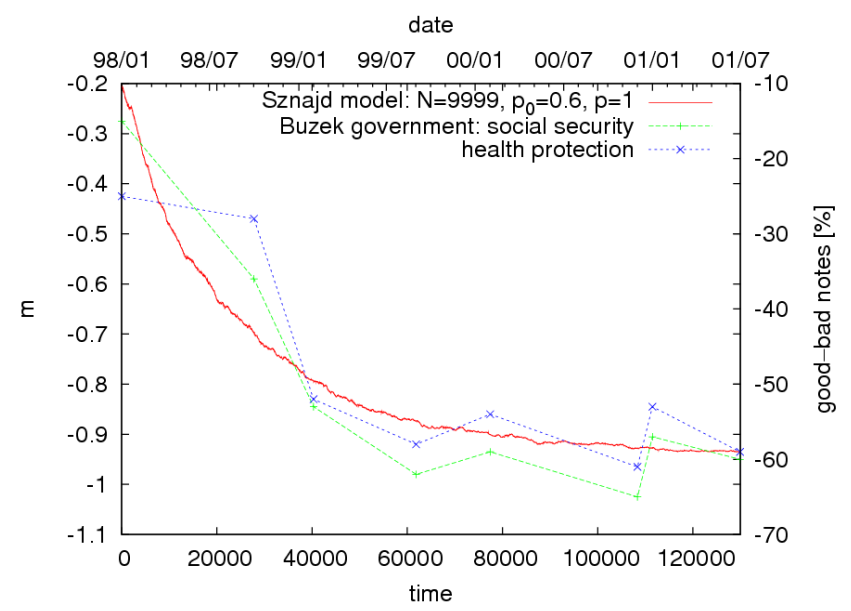

Fig. 5. The fall of the public support for the policy of the government of Jerzy Buzek on the social issues and the time evolution of the main opinion in the Sznajd model for $N=9999, p_{0}=0.6$ and $p=1$. 
The linear dependence of the time $\tau$ on the system size $N$ is the same as found analytically for the fully connected graph [23]. On the other hand, the theoretical prediction [23] of the $\tau$ dependence on $p_{0}$ is not confirmed by our simulations. This means that the result $\tau \propto N$ is valid for a larger class of networks.

In our simulations, all nodes are selected at each time step, in different order, and the Sznajd rule is executed with probability $p$. The $\tau$ dependence on $p$ is slower than just $1 / p$; the latter could be expected if the decrease in $p$ is equivalent just to slowing the process down. The difference can be due to the fact that once $p<1$, some nodes are not updated at a given time step. Paradoxically, the presence of these temporal "contrarians" seems to accelerate the process of getting stationary state.

The obtained distribution of $\tau$ is close to the log-normal distribution, in accordance with the discussion in Ref. [24]. This means that the phase transition is well defined. The distribution of times between subsequent changes of state of a given node is shown in Fig. 4b. The plot is different from the scaling $\Delta t^{-3 / 2}$, obtained in Ref. [12] for the one-dimensional system.

Once $p=1$, the distribution of time between subsequent picking up of the same node (irrespectively whether it is changed or not) is the tent function, with the average $T=N$. In the case when the nodes are selected randomly and not as noted above, the same distribution is the exponential one, as in Fig. 4c.

Concluding, the Sznajd dynamics has been applied to the directed network designed as to reproduce the small world effect and clusterization, as in social networks. The results on the time $\tau$ of getting stationary state are compared to the analytical calculations for the fully connected network [23]. Although the dependence of $\tau$ on the initial state is different than in theory, the system size dependence of $\tau$ is the same.

The message for the social applications is that the time of attaining the stationary distribution increases linearly with the system size. Among other consequences, this means that an initial support for a new government persists twice longer in a twice larger country; larger countries have more time for reforms. Still, even if the initial state is the longest-lived $p_{0}=0.5$, this time is not infinite, as it is shown in Fig. 3a. As remarked in Sect. 1, all that is valid as long as opinions are adjusted in small groups. Important political events can reveal a metastable character of the opinion distribution; however, this possibility remains out of frames of this work.

\section{Acknowledgments}

The authors are grateful to Janusz Hołyst for his critical comments. The numerical calculations were carried out in ACK-CYFRONET-AGH. The machine time on HP Integrity Superdome is financed by the Polish Ministry of Science and Higher Education under grant No. MNiI/HP_I_SDAGH/047/2004. 


\section{References}

[1] Woodrow Wilson, the speech to newspapers editors and publishers at the Waldorf-Astoria, 20 April 1915.

[2] W. Lippmann, Public Opinion, 1922; Free Press, New York 1997.

[3] J. Shamir, M. Shamir, The Anatomy of Public Opinion, University of Michigan Press, Ann Arbor 2000.

[4] S. Wasserman, K. Faust, Social Network Analysis: Methods and Applications, Cambridge UP, Cambridge 1997.

[5] A. Campbell, P.E. Converse, W.E. Miller, D.E. Stokes, The American Voter, Wiley, Chicago 1960.

[6] E. Noelle-Neumann, The Spiral of Silence: Public Opinion - Our Social Skin, Univ. of Chicago, Chicago 1984.

[7] D. Stauffer, arXiv:0705.0891.

[8] C. Castellano, S. Fortunato, V. Loreto, arXiv:0710.3256.

[9] M. Schnegg, Int. J. Mod. Phys. C 17, 1067 (2006).

[10] P. Clifford, A. Sudbury, Biometrika 60, 581 (1973).

[11] S. Redner, A Guide to First-Passage Processes, Cambridge UP, Cambridge 2001.

[12] K. Sznajd-Weron, J. Sznajd, Int. J. Mod. Phys. C 11, 1157 (2000).

[13] D. Stauffer, J. Artificial Society Social Simul. 5, issue 1, paper 4 (2001), http://jasss.soc.surrey.ac.uk/5/1/4.html.

[14] D. Stauffer, S. Moss de Oliveira, P.M.C. de Oliveira, J.S.Sá Martins, Biology, Sociology, Geology by Computational Physicists, Elsevier, Amsterdam 2006.

[15] G. Deffuant, D. Neau, F. Amblard, G. Weisbuch, in: Applications of Simulation to Social Science, Eds. G. Ballot, G. Weisbuch, Editions Hermes Sciences, Oxford 2000.

[16] M. Granovetter, Am. J. Soc. 83, 1420 (1978).

[17] D.J. Watts, S.H. Strogatz, Nature 393, 440 (1998).

[18] M.C. González, H.J. Herrmann, J. Kertész, T. Vicsek, Physica A 379, 307 (2007).

[19] Public Opinion Research Center, Report BS/145/2007 (in Polish) (http://www.cbos.pl/).

[20] P. Erdős, A. Rényi, Publications Mathematicae 6, 290 (1959); P. Erdős, A. Rényi, Publ. Math. Inst. Hung. Acad. Sci. 5, 17 (1960).

[21] P. Holme, B.J. Kim, Phys. Rev. E 65, 026107 (2002).

[22] M.A. Serrano, M. Boguna, Phys. Rev. E 72, 036133 (2005).

[23] F. Slanina, H. Lavicka, Eur. Phys. J. B 35, 279 (2003).

[24] D. Stauffer, A.O. Sousa, S. Moss de Oliveira, Int. J. Mod. Phys. C 11, 1239 (2000). 